Research Article

\title{
European Spread Option Pricing with the Floating Interest Rate for Uncertain Financial Market
}

\author{
Lidong Zhang $\mathbb{D}^{1,2}$ Yanmei Sun, ${ }^{3}$ and Xiangbo Meng ${ }^{1}$ \\ ${ }^{1}$ School of Science, Tianjin University of Science \& Technology, Tianjin 300457, China \\ ${ }^{2}$ Center for Financial Engineering and Risk Management, Tianjin University of Science \& Technology, Tianjin 300222, China \\ ${ }^{3}$ College of Economics \& Management, Tianjin University of Science \& Technology, Tianjin 300222, China
}

Correspondence should be addressed to Lidong Zhang; zhanglidong1999@126.com

Received 15 April 2020; Accepted 5 May 2020; Published 21 May 2020

Guest Editor: Wenguang Yu

Copyright ( 92020 Lidong Zhang et al. This is an open access article distributed under the Creative Commons Attribution License, which permits unrestricted use, distribution, and reproduction in any medium, provided the original work is properly cited.

In this paper, we investigate the pricing problems of European spread options with the floating interest rate. In this model, uncertain differential equation and stochastic differential equation are used to describe the fluctuation of stock price and the floating interest rate, respectively. We derive the pricing formulas for spread options including the European spread call option and the European spread put option. Finally, numerical algorithms are provided to illustrate our results.

\section{Introduction}

Since financial derivatives have the function of hedging and risk aversion, they are widely used in the financial market. Options are special financial derivatives, and they are used not only in the adjustment of the national debt but also in the leveraged investment of enterprises and the hedging of commodities. The value of an option depends not only on the price of its underlying asset but also on other factors such as the interest rate. Since Black and Scholes [1] established the B-S model, option pricing has become an important issue in financial mathematical research.

However, the expected return of a stock is different from the assumption of the B-S model in the financial market. In order to better match with the real market, scholars have improved the B-S model. Merton [2] proposed a jump-diffusion model and gave the European option pricing formula. Hull and White [3] studied the pricing problem for the European option under stochastic volatility. Heston [4] investigated the option pricing under stochastic interest rate and studied the pricing problems of the bond and currency option. Stock prices are easily affected by the social environment and investor belief degrees, but the existing models and theories are difficult to give a reasonable explanation. For dealing with the uncertainty of human behavior, Liu [5] founded the uncertainty theory based on normality, duality, subadditivity, and product axioms. To describe uncertain dynamic systems, Liu [6] introduced the concept of uncertain processes and proposed uncertain differential equations driven by canonical Liu process. Liu [7] proposed an uncertain stock model in which stock price was described by an uncertain differential equation. Subsequently, Chen [8] and Zhang and Liu [9] gave the pricing formulas for the European option, American option, and geometric Asian option, respectively. Considering the uncertain fluctuations of the interest rate, Chen and Gao [10] firstly studied the term structure of the uncertain interest rate. Yao [11] proposed an uncertain stock model with floating interest rate. Zhang et al. [12] derived the pricing formulas of interest rate ceiling and interest rate floor. Sun and Su [13] studied the pricing problems for the European and American option under the mean-reverting stock model. Gao et al. [14] discussed the pricing formulas of lookback options based on the uncertain exponential Ornstein-Uhlenbeck model. The latest research on the applications of uncertainty theory and probability theory was given by Gao [15], Lu and Zhu [16], Li et al. [17], Yu et al. $[18,19]$, and Zhang and Sun [20].

In this paper, we investigate the pricing problems for European spread options. It is well known that spread 
options are path-dependent exotic options whose returns depend on the spread of two or more assets. Although spread options are widely traded in different financial markets, it is still difficult to price such options. At present, there are relatively few research studies on the pricing of spread options. It is well known that interest rate is an important factor influencing the price of financial derivatives, and it is often fluctuated by stochastic factors such as economy and policy. Two stocks issued newly are considered in this paper, so the lack of historical data leads to the unsuccessful investigation on the price of spread options based on probability theory. Thus, basing uncertainty theory and probability theory (socalled chance theory), we study the pricing of spread options with a stochastic interest rate under uncertain environment. We introduce some basic knowledge of uncertainty theory and chance theory in Section 2. Then, we derive European spread call option and put option pricing formulas with stochastic interest rates and also give some numerical algorithms to calculate the prices in Section 3. Finally, a brief conclusion is given in Section 4 .

\section{Preliminaries}

This section mainly introduces uncertainty theory and chance theory. Uncertainty theory is an effective tool for dealing with reliability issues related to human uncertainty, while chance theory is a basic tool to deal with complex systems with randomness and uncertainty. This section provides some basic definitions and results on uncertainty theory and chance theory. For more details, please see Liu's latest book [21].

\subsection{Uncertainty Theory}

\subsubsection{Uncertain Variable}

Definition 1 (see Liu $[5,7]$ ). Let $L$ be a $\sigma$-algebra on a nonempty set $\Gamma$. A set function $M: L \longrightarrow[0,1]$ is called an uncertain measure if it satisfies the following axioms:

Axiom 1 (normality axiom): $M\{\Gamma\}=1$ for the universal set $\Gamma$.

Axiom 2 (duality axiom): $M\{\Lambda\}+M\left\{\Lambda^{c}\right\}=1$ for any event $\Lambda$.

Axiom 3 (subadditivity axiom): for every countable sequence of events $\Lambda_{1}, \Lambda_{2}, \ldots$, we have

$$
M\left\{\bigcup_{i=1}^{\infty} \Lambda_{i}\right\} \leq \sum_{i=1}^{\infty} M\left\{\Lambda_{i}\right\}
$$

Axiom 4 (product axiom): let $\left(\Gamma_{k}, L_{k}, M_{k}\right)$ be uncertainty spaces for $k=1,2, \ldots$. The product uncertain measure $M$ is an uncertain measure satisfying

$$
M\left\{\prod_{k=1}^{\infty} \Lambda_{k}\right\}=\bigwedge_{k=1}^{\infty} M_{k}\left\{\Lambda_{k}\right\},
$$

where $\Lambda_{k}$ are arbitrarily chosen events from $L_{k}$ for $k=1,2, \ldots$, respectively.

Definition 2 (see Liu [5]). An uncertain variable is a function from an uncertainty space $(\Gamma, L, M)$ to the set of real numbers; for any Borel set $B$ of real numbers, the set

$$
\{\xi \in B\}=\{\gamma \in \Gamma \mid \xi(\gamma) \in B\}
$$

is an event.

The uncertainty distribution $\Phi(x)$ of an uncertain variable $\xi$ is defined by $\Phi(x)=M\{\xi \leq x\}$ for any real number $x$. An uncertainty distribution $\Phi(x)$ is said to be regular if it is a continuous and strictly increasing function with respect to $x$ at which $0<\Phi(x)<1$, and

$$
\begin{aligned}
& \lim _{x \longrightarrow-\infty} \Phi(x)=0, \\
& \lim _{x \longrightarrow+\infty} \Phi(x)=1 .
\end{aligned}
$$

If $\xi$ has a regular uncertainty distribution $\Phi(x)$, then the inverse function $\Phi^{-1}(\alpha)$ is called the inverse uncertainty distribution of $\xi$.

Definition 3 (see Liu [7]). The uncertain variables $\xi_{1}, \xi_{2}, \ldots, \xi_{m}$ are said to be independent if

$$
M\left\{\bigcap_{i=1}^{m}\left\{\xi_{i} \in B_{i}\right\}\right\}=\bigwedge_{i=1}^{m} M\left\{\xi_{i} \in B_{i}\right\},
$$

for any Borel sets $B_{1}, B_{2}, \ldots, B_{m}$ of real numbers.

Liu [22] proposed the operation law of uncertain variables and calculated the inverse uncertainty distribution of strictly monotone function of uncertain variables.

Theorem 1 (see Liu [22]). Let $\xi_{1}, \xi_{2}, \ldots, \xi_{n}$ be independent uncertain variables with uncertainty distributions $\Phi_{1}, \Phi_{2}, \ldots, \Phi_{n}$. If the function $f\left(x_{1}, x_{2}, \ldots, x_{n}\right)$ is strictly increasing with respect to $x_{1}, x_{2}, \ldots, x_{m}$ and strictly decreasing with $x_{m+1}, x_{m+2}, \ldots, x_{n}$, then

$$
\xi=f\left(\xi_{1}, \xi_{2}, \ldots, \xi_{m}, \xi_{m+1}, \xi_{m+2}, \ldots, \xi_{n}\right),
$$

is an uncertain variable with inverse uncertainty distribution $\Psi^{-1}(\alpha)=f\left(\Phi_{1}^{-1}(\alpha), \ldots, \Phi_{m}^{-1}(\alpha), \Phi_{m+1}^{-1}(1-\alpha), \ldots, \Phi_{n}^{-1}(1-\alpha)\right)$.

Definition 4 (see Liu [5]). The expected value of an uncertain variable $\xi$ is defined by

$$
E[\xi]=\int_{0}^{+\infty} M\{\xi \geq x\} \mathrm{d} x-\int_{-\infty}^{0} M\{\xi \leq x\} \mathrm{d} x,
$$

provided that at least one of the two integrals exists.

For an uncertain variable $\xi$ with an uncertainty distribution $\Phi(x)$, if its expected value exists, Liu [5] showed that

$$
E[\xi]=\int_{0}^{+\infty}(1-\Phi(x)) \mathrm{d} x-\int_{-\infty}^{0} \Phi(x) \mathrm{d} x .
$$


Theorem 2 (see Liu [22]). Assume the uncertain variable $\xi$ has a regular uncertainty distribution $\Phi$; then,

$$
E[\xi]=\int_{0}^{1} \Phi^{-1}(\alpha) \mathrm{d} \alpha .
$$

2.1.2. Uncertain Differential Equations. Liu [6] proposed the concept of uncertain process and defined the time integral of uncertain process.

Definition 5 (see Liu [6]). Let $T$ be an index set, and let $(\Gamma, L, M)$ be an uncertainty space. An uncertain process is a measurable function from $T \times(\Gamma, L, M)$ to the set of real numbers; for each $t \in T$ and any Borel set $B$,

$$
\left\{X_{t} \in B\right\}=\left\{\gamma \in \Gamma \mid X_{t}(\gamma) \in B\right\},
$$

is an event.

An uncertain process $X_{t}$ is said to have independent increments if $X_{t_{0}}, X_{t_{1}}-X_{t_{0}}, X_{t_{2}}-X_{t_{1}}, \ldots, X_{t_{k}}-X_{t_{k-1}}$ are independent uncertain variables, where $t_{0}$ is the initial time and $t_{1}, t_{2}, \ldots, t_{k}$ are any times with $t_{0}<t_{1}<\cdots<t_{k}$. An uncertain process $X_{t}$ is said to have stationary increments if for any given $t>0$, the increments $X_{s+t}-X_{s}$ are identically distributed uncertain variables for all $s>0$.

Definition 6 (see Liu [7]). An uncertain process $C_{t}$ is said to be a Liu process if

(i) $C_{t}=0$, and almost all sample paths are Lipschitz continuous.

(ii) $C_{t}$ has stationary and independent increments.

(iii) Every increment $C_{s+t}-C_{s}$ is a normal uncertain variable with expected value 0 and variance $t^{2}$, whose uncertainty distribution is

$$
\Phi_{t}(x)=\left(1+\exp \left(\frac{-\pi x}{\sqrt{3} t}\right)\right)^{-1}, \quad x \in \Re
$$

Definition 7 (see Liu [5]). Let $X_{t}$ be an uncertain process, and let $C_{t}$ be a Liu process. For any partition of closed interval $[a, b]$ with $a=t_{1}<t_{2}<\cdots<t_{k+1}=b$, the mesh is written as

$$
\Delta=\max _{1 \leq i \leq k}\left|t_{i+1}-t_{i}\right| .
$$

Then, Liu integral of $X_{t}$ with respect to $C_{t}$ is defined as

$$
\int_{a}^{b} X_{t} \mathrm{~d} C_{t}=\lim _{\Delta \longrightarrow 0} \sum_{i=1}^{k} X_{t} \cdot\left(C_{t_{i+1}}-C_{t_{i}}\right),
$$

provided that the limit exists almost surely and is finite. In this case, the uncertain process is said to be integrable.

Definition 8 (see Liu [6]). Suppose $C_{t}$ is a Liu process, and $f$ and $g$ are two functions. Then,

$$
\mathrm{d} X_{t}=f\left(t, X_{t}\right) \mathrm{d} t+g\left(t, X_{t}\right) \mathrm{d} C_{t},
$$

is called an uncertain differential equation. A solution is an uncertain process $X_{t}$ that satisfies the equation identically in $t$.

Definition 9 (see Yao and Chen [23]). Let $\alpha$ be a number with $0<\alpha<1$. An uncertain differential equation

$$
\mathrm{d} X_{t}=f\left(t, X_{t}\right) \mathrm{d} t+g\left(t, X_{t}\right) \mathrm{d} C_{t},
$$

is said to have an $\alpha$-path $X_{t}^{\alpha}$ if it solves the corresponding ordinary differential equation:

$$
\mathrm{d} X_{t}^{\alpha}=f\left(t, X_{t}^{\alpha}\right) \mathrm{d} t+\left|g\left(t, X_{t}^{\alpha}\right)\right| \Phi^{-1}(\alpha) \mathrm{d} t,
$$

where $\Phi^{-1}(\alpha)$ is the inverse uncertainty distribution of the standard normal uncertain variable

$$
\Phi^{-1}(\alpha)=\frac{\sqrt{3}}{\pi} \ln \frac{\alpha}{1-\alpha} .
$$

Theorem 3 (see Yao and Chen [23]). Let $X_{t}$ and $X_{t}^{\alpha}$ be the solution and $\alpha$-path of the uncertain differential equation

$$
\mathrm{d} X_{t}=f\left(t, X_{t}\right) \mathrm{d} t+g\left(t, X_{t}\right) \mathrm{d} C_{t},
$$

respectively. Then,

$$
\begin{aligned}
& M\left\{X_{t} \leq X_{t}^{\alpha}, \forall t\right\}=\alpha, \\
& M\left\{X_{t}>X_{t}^{\alpha}, \forall t\right\}=1-\alpha .
\end{aligned}
$$

\subsection{Chance Theory}

Definition 10. Let $(\Gamma, L, M)$ be an uncertainty space, and let $(\Omega, \mathscr{F}, \mathrm{P})$ be a probability space. Then, the product $(\Gamma, L, M) \times(\Omega, \mathscr{F}, P)$ is called a chance space.

Definition 11 (see Liu [24]). Let $(\Gamma, L, M) \times(\Omega, \mathscr{F}, P)$ be a chance space, and let $\Theta \in L \times \mathscr{F}$ be an event. Then, the chance measure of $\Theta$ is defined as

$$
\operatorname{Ch}\{\Theta\}=\int_{0}^{1} P\{\omega \in \Omega \mid M\{\gamma \in \Gamma \mid(\gamma, \omega) \in \Theta\} \geq x\} \mathrm{d} x .
$$

Theorem 4 (see Liu [24]). Let $\xi$ be an uncertain random variable on the chance space $(\Gamma, L, M) \times(\Omega, \mathscr{F}, P)$, and let $B$ be a Borel set of real numbers. Then, $\{\xi \in B\}$ is an uncertain random event with chance measure

$$
C h\{\xi \in B\}=\int_{0}^{1} P\{\omega \in \Omega \mid M\{\gamma \in \Gamma \mid(\gamma, \omega) \in B\} \geq x\} \mathrm{d} x .
$$

Definition 12 (see Liu [24]). Let $\xi$ be an uncertain random variable. Then, its chance distribution is defined by

$$
\Phi(x)=\operatorname{Ch}\{\xi \leq x\},
$$

for any $x \in R$. 
Theorem 5 (see Liu [25]). Let $\eta_{1}, \eta_{2}, \ldots, \eta_{m}$ be independent random variables with probability distributions $\Psi_{1}, \Psi_{2}, \ldots, \Psi_{m}$, and let $\tau_{1}, \tau_{2}, \ldots, \tau_{n}$ be independent uncertain variables with uncertainty distributions $\Upsilon_{1}, \Upsilon_{2}, \ldots, \Upsilon_{n}$, respectively. Iff is a measurable function, then the uncertain random variable

$$
\xi=f\left(\eta_{1}, \eta_{2}, \ldots, \eta_{m}, \tau_{1}, \tau_{2}, \ldots, \tau_{n}\right),
$$

has a chance distribution

$$
\Phi(x)=\int_{R^{m}} F\left(x ; y_{1}, y_{2}, \ldots, y_{m}\right) \mathrm{d} \Psi_{1}\left(y_{1}\right) \mathrm{d} \Psi_{2}\left(y_{2}\right) \ldots \mathrm{d} \Psi_{m}\left(y_{m}\right),
$$

where

$$
F\left(x ; y_{1}, y_{2}, \ldots, y_{m}\right)=M\left\{f\left(y_{1}, y_{2}, \ldots, y_{m}, \tau_{1}, \tau_{2}, \ldots, \tau_{n}\right) \leq x\right\}
$$

is the uncertainty distribution of $f\left(y_{1}, y_{2}, \ldots, y_{m}, \tau_{1}\right.$, $\left.\tau_{2}, \ldots, \tau_{n}\right)$ for any real numbers $y_{1}, y_{2}, \ldots, y_{m}$ and is determined by $\Upsilon_{1}, \Upsilon_{2}, \ldots, \Upsilon_{n}$.

Definition 13 (see Liu [24]). Let $\xi$ be an uncertain random variable. Then, its expected value is defined by

$$
E[\xi]=\int_{0}^{+\infty} C h\{\xi \geq x\} \mathrm{d} x-\int_{-\infty}^{0} C h\{\xi \leq x\} \mathrm{d} x,
$$

provided that at least one of the two integrals is finite.

Theorem 6 (see Liu [24]). Let $\xi$ be an uncertain random variable with chance distribution $\Phi$. Then,

$$
E[\xi]=\int_{-\infty}^{+\infty} x \mathrm{~d} \Phi(x) .
$$

Theorem 7 (see Liu [25]). Let $\eta_{1}, \eta_{2}, \ldots, \eta_{m}$ be independent random variables with probability distributions $\Psi_{1}, \Psi_{2}, \ldots, \Psi_{m}$, and let $\tau_{1}, \tau_{2}, \ldots, \tau_{n}$ be independent uncertain variables with uncertainty distributions $\Upsilon_{1}, \Upsilon_{2}, \ldots, \Upsilon_{n}$, respectively. Iff is a measurable function, then the uncertain random variable

$$
\xi=f\left(\eta_{1}, \eta_{2}, \ldots, \eta_{m}, \tau_{1}, \tau_{2}, \ldots, \tau_{n}\right)
$$

has an expected value

$$
E(\xi)=\int_{R^{m}} G\left(y_{1}, y_{2}, \ldots, y_{m}\right) \mathrm{d} \Psi_{1}\left(y_{1}\right) \mathrm{d} \Psi_{2}\left(y_{2}\right) \ldots \mathrm{d} \Psi_{m}\left(y_{m}\right),
$$

where

$$
G\left(y_{1}, y_{2}, \ldots, y_{m}\right)=E\left[f\left(y_{1}, y_{2}, \ldots, y_{m}, \tau_{1}, \tau_{2}, \ldots, \tau_{n}\right)\right]
$$

is the uncertainty distribution of $f\left(y_{1}, y_{2}, \ldots, y_{m}, \tau_{1}\right.$, $\left.\tau_{2}, \ldots, \tau_{n}\right)$ for any real numbers $y_{1}, y_{2}, \ldots, y_{m}$ and is determined by $\Upsilon_{1}, \Upsilon_{2}, \ldots, \Upsilon_{n}$.

Corollary 1. Let $\eta_{1}, \eta_{2}, \ldots, \eta_{m}$ be independent random variables with probability distributions $\Psi_{1}, \Psi_{2}, \ldots, \Psi_{m}$, and let $\tau_{1}, \tau_{2}, \ldots, \tau_{n}$ be independent uncertain variables with uncertainty distributions $\Upsilon_{1}, \Upsilon_{2}, \ldots, \Upsilon_{n}$, respectively. If $f$ and $g$ are measurable functions, then the uncertain random variable

$$
\xi=f\left(\eta_{1}, \eta_{2}, \ldots, \eta_{m}\right) \times g\left(\tau_{1}, \tau_{2}, \ldots, \tau_{n}\right),
$$

has an expected value

$$
E(\xi)=E\left[f\left(\eta_{1}, \eta_{2}, \ldots, \eta_{m}\right)\right] \times E\left[g\left(\tau_{1}, \tau_{2}, \ldots, \tau_{n}\right)\right] .
$$

Proof. From Theorem 7, the expectation of the uncertain random variable $\xi$ can be given by the following equation:

$$
\begin{aligned}
E(\xi)= & \int_{R^{m}} E\left[f\left(y_{1}, y_{2}, \ldots, y_{m}\right) \times g\left(\tau_{1}, \tau_{2}, \ldots, \tau_{n}\right)\right] \\
& \mathrm{d} \Psi_{1}\left(y_{1}\right) \mathrm{d} \Psi_{2}\left(y_{2}\right) \ldots \mathrm{d} \Psi_{m}\left(y_{m}\right) \\
= & \int_{R^{m}} f\left(y_{1}, y_{2}, \ldots, y_{m}\right) \times E\left[g\left(\tau_{1}, \tau_{2}, \ldots, \tau_{n}\right)\right] \\
& \mathrm{d} \Psi_{1}\left(y_{1}\right) \mathrm{d} \Psi_{2}\left(y_{2}\right) \ldots \mathrm{d} \Psi_{m}\left(y_{m}\right) \\
= & \int_{R^{m}} f\left(y_{1}, y_{2}, \ldots, y_{m}\right) \mathrm{d} \Psi_{1}\left(y_{1}\right) \mathrm{d} \Psi_{2}\left(y_{2}\right) \ldots \mathrm{d} \Psi_{m}\left(y_{m}\right) \\
& \times E\left[g\left(\tau_{1}, \tau_{2}, \ldots, \tau_{n}\right)\right] \\
= & E\left[f\left(\eta_{1}, \eta_{2}, \ldots, \eta_{m}\right)\right] \times E\left[g\left(\tau_{1}, \tau_{2}, \ldots, \tau_{n}\right)\right] .
\end{aligned}
$$

\section{European Spread Option Pricing Formulas}

In this section, we discuss the pricing of spread options under the stochastic interest rate environment where stock prices follow uncertain exponential Ornstein-Uhlenbeck process and uncertain log-normal process, respectively. Firstly, we assume interest rate $r$, one stock price $X$, and another stock price $Y$ which satisfy the following differential equation, respectively:

$$
\left\{\begin{array}{l}
\mathrm{d} r_{t}=a\left(b-r_{t}\right) \mathrm{d} t+\sigma_{0} \sqrt{r_{t}} \mathrm{~d} W_{t}, \\
\mathrm{~d} X_{t}=\mu_{1}\left(1-c \ln X_{t}\right) X_{t} \mathrm{~d} t+\sigma_{1} X_{t} \mathrm{~d} C_{1 t}, \\
\mathrm{~d} Y_{t}=\mu_{2} Y_{t} \mathrm{~d} t+\sigma_{2} Y_{t} \mathrm{~d} C_{2 t},
\end{array}\right.
$$

where $a, b, \sigma_{0}, \mu_{1}, c, \sigma_{1}, \mu_{2}, \sigma_{2}$ are some positive real numbers, $W_{t}$ is a Brownian motion, and $C_{1 t}$ and $C_{2 t}$ are independent canonical Liu processes.

Then, the inverse uncertainty distribution of $X_{t}, Y_{t}$ is given by Dai et al. [26] and Yao and Chen [23]. The specific form is as follows:

$$
\begin{aligned}
X_{t}^{-1}(\alpha)= & \exp \left(\exp \left(-\mu_{1} c t\right) \ln X_{0}+\frac{1-\exp \left(-\mu_{1} c t\right)}{c}\right. \\
& \left.\left(1+\frac{\sqrt{3} \sigma_{1}}{\mu_{1} \pi} \ln \frac{\alpha}{1-\alpha}\right)\right), \\
Y_{t}^{-1}(\alpha)= & Y_{0} \exp \left(\mu_{2} t+\frac{\sqrt{3} \sigma_{2}}{\pi} t \ln \frac{\alpha}{1-\alpha}\right) .
\end{aligned}
$$


In the next sections, the pricing formulas of the European spread call option and the European spread put option are derived.

3.1. European Spread Call Option. Suppose $X_{t}$ and $Y_{t}$ are two asset price processes. Then, the European spread call option with maturity date $T$ and strike price $K$ is the contract that pays

$$
\left(X_{T}-Y_{T}-K\right)^{+}
$$

Considering time value of the stock return, the present value of the payoff is

$$
\exp \left(-\int_{0}^{T} r_{t} \mathrm{~d} t\right)\left(X_{T}-Y_{T}-K\right)^{+}
$$

European spread call option should be the expectation of the discounted value of the stock return. So, the European spread call option has a price

$$
f_{\text {Ecall }}=E\left[\exp \left(-\int_{0}^{T} r_{t} \mathrm{~d} t\right)\left(X_{T}-Y_{T}-K\right)^{+}\right] .
$$

Theorem 8. Suppose that the European spread call option for the stock model (35) has a strike price $K$ and an expiration time $T$. Then, the European spread call option pricing formula is

$$
f_{\text {Ecall }}=E\left[\exp \left(-\int_{0}^{T} r_{t} \mathrm{~d} t\right)\right] \int_{0}^{1}\left(X_{T}^{-1}(\alpha)-Y_{T}^{-1}(1-\alpha)-K\right)^{+} \mathrm{d} \alpha .
$$

Proof. According to Corollary 1, European spread call option is given by the following form:

$$
f_{\text {Ecall }}=E\left[\exp \left(-\int_{0}^{T} r_{t} \mathrm{~d} t\right)\right] E\left[\left(X_{T}-Y_{T}-K\right)^{+}\right]
$$

Since $X_{t}, Y_{t}$ are independent uncertain processes, their $\alpha$-path are, respectively, given by formulas (36) and (37). According to Theorem 1, the value of investor's return difference at the maturity date $T$,

$$
\left(X_{T}-Y_{T}-K\right)^{+}
$$

has the $\alpha$-path

$$
\left(X_{T}^{-1}(\alpha)-Y_{T}^{-1}(1-\alpha)-K\right)^{+}
$$

So, the expectation of the uncertain variable $\left(X_{T}-Y_{T}-K\right)^{+}$can be derived easily:

$$
E\left[\left(X_{T}-Y_{T}-K\right)^{+}\right]=\int_{0}^{1}\left(X_{T}^{-1}(\alpha)-Y_{T}^{-1}(1-\alpha)-K\right)^{+} \mathrm{d} \alpha .
$$

Thus, the price of the European spread call option is $f_{\text {Ecall }}=E\left[\exp \left(-\int_{0}^{T} r_{t} \mathrm{~d} t\right)\right] \int_{0}^{1}\left(X_{T}^{-1}(\alpha)-Y_{T}^{-1}(1-\alpha)-K\right)^{+} \mathrm{d} \alpha$.

The pricing formula of the European spread call option is derived.

From Theorem 8, the algorithm designed for calculating European spread call option price is divided by two parts. In the first procedure, $E\left[\exp \left(-\int_{0}^{T} r_{t} \mathrm{~d} t\right)\right]$ is calculated by Monte Carlo simulation. In the second procedure, $E\left[\left(X_{T}-Y_{T}-K\right)^{+}\right]$can be calculated by using the property of inverse distribution. In the final procedure, European spread call option price is derived by virtue of Theorem 8 .

In the first procedure, we will calculate $E\left[\exp \left(-\int_{0}^{T} r_{t} \mathrm{~d} t\right)\right]$. We will first generate some sample trajectories by stochastic simulations and calculate the average of the terminal trajectories.

Step 0: set $t_{j}=j T / M, k=1,2, \ldots, L, j=1,2, \ldots, M$, where $L$ and $M$ are two large numbers.

Step 1: set $k=0$.

Step 2: set $k \longleftarrow k+1$.

Step 3: set $j=0$.

Step 4: set $j \longleftarrow j+1$.

Step 5: calculate the value of the stochastic interest rate at the time $t_{j}$ :

$$
r_{t_{j}}^{k}=r_{t_{j-1}}^{k}+a\left(b-r_{t_{j-1}}^{k}\right)\left(t_{j}-t_{j-1}\right)+\sigma_{0} \sqrt{r_{t_{j-1}}^{k}} \sqrt{t_{j}-t_{j-1}} \varepsilon_{j}^{k},
$$

where $\varepsilon_{j}^{k} \sim N(0,1)$ are generated by stochastic simulations.

Step 6: calculate the discount rate:

$$
\exp \left(-\int_{0}^{T} r_{t}^{k} \mathrm{~d} t\right) \longleftarrow \exp \left(-\frac{T}{M} \sum_{j=1}^{M} r_{t_{j}}^{k}\right)
$$

Step 7: calculate the approximated value of $E\left[\exp \left(-\int_{0}^{T} r_{t} \mathrm{~d} t\right)\right]$ :

$$
E\left[\exp \left(-\int_{0}^{T} r_{t} \mathrm{~d} t\right)\right] \longleftarrow \frac{1}{L} \sum_{k=1}^{L} \exp \left(-\frac{T}{M} \sum_{j=1}^{M} r_{t_{j}}^{k}\right)
$$

In the second procedure, we will calculate $E\left[\left(X_{T}-Y_{T}-K\right)^{+}\right]$.

Step 0: set $\alpha_{i}=i / N, i=1,2, \ldots, N-1$, where $N$ is a large number.

Step 1: set $i=0$.

Step 2: set $i \longleftarrow i+1$.

Step 3: calculate the inverse uncertainty distribution of the stock processes: 


$$
\begin{aligned}
X_{T}^{-1}\left(\alpha_{i}\right)= & \exp \left(\exp \left(-\mu_{1} c T\right) \ln X_{0}+\frac{1}{c}\left(1-\exp \left(-\mu_{1} c T\right)\right)\right. \\
& \left.\left(1+\frac{\sqrt{3} \sigma_{1}}{\mu_{1} \pi} \ln \frac{\alpha_{i}}{1-\alpha_{i}}\right)\right), \\
Y_{T}^{-1}\left(1-\alpha_{i}\right)= & Y_{0} \times \exp \left(\mu_{2} T+\frac{\sqrt{3} \sigma_{2}}{\pi} T \ln \frac{1-\alpha_{i}}{\alpha_{i}}\right) .
\end{aligned}
$$

Step 4: set

$$
\beta^{\alpha_{i}} \longleftarrow \max \left(X_{T}^{-1}\left(\alpha_{i}\right)-Y_{T}^{-1}\left(1-\alpha_{i}\right)-K, 0\right) .
$$

If $i<N-1$, then return to Step 2 .

Step 5: calculate the expectation of $\left(X_{T}-Y_{T}-K\right)^{+}$:

$$
E\left[\left(X_{T}-Y_{T}-K\right)^{+}\right] \longleftarrow \frac{1}{N-1} \sum_{i=1}^{N-1} \beta^{\alpha_{i}} .
$$

In the final procedure, the price of the European spread call option is derived by using Theorem 8 .

Example 1. Assume that the parameters of interest rate are $r_{0}=0.08, a=0.05, \sigma_{0}=0.04, b=2$, the parameters of stock price $X$ are $X_{0}=5, \mu_{1}=\sqrt{3}, \sigma_{1}=0.3, c=0.3$, and the parameters of stock price $Y$ are $Y_{0}=4, \mu_{2}=1, \sigma_{2}=0.2$. Then, the price of the European spread call option with maturity date $T=1$ and strike price $K=1$ is $f_{E \text { call }}=2.0372$.

3.2. European Spread Put Option. Suppose $X_{t}$ and $Y_{t}$ are two asset price processes. Then, the European spread put option with maturity date $T$ and strike price $K$ is the contract that pays

$$
\left(K-\left(X_{T}-Y_{T}\right)\right)^{+} \text {. }
$$

Considering time value of the stock return, the present value of the payoff is

$$
\exp \left(-\int_{0}^{T} r_{t} \mathrm{~d} t\right)\left(K-\left(X_{T}-Y_{T}\right)\right)^{+}
$$

European spread put option should be the expectation of the discounted value of the stock return. So, the European spread put option has a price

$$
f_{E \text { put }}=E\left[\exp \left(-\int_{0}^{T} r_{t} \mathrm{~d} t\right)\left(K-\left(X_{T}-Y_{T}\right)\right)^{+}\right] .
$$

Theorem 9. Suppose that the European spread put option for the stock model (35) has a strike price $K$ and an expiration time $T$. Then, the European spread put option pricing formula is

$$
f_{\text {Eput }}=E\left[\exp \left(-\int_{0}^{T} r_{t} \mathrm{~d} t\right)\right] \int_{0}^{1}\left(K-\left(X_{T}^{-1}(1-\alpha)-Y_{T}^{-1}(\alpha)\right)\right)^{+} \mathrm{d} \alpha .
$$

Proof. According to Corollary 1, the European spread put option can be given in the following form:

$$
f_{\text {Eput }}=E\left[\exp \left(-\int_{0}^{T} r_{t} \mathrm{~d} t\right)\right] E\left[\left(K-\left(X_{T}-Y_{T}\right)\right)^{+}\right]
$$

Since $X_{t}, Y_{t}$ are independent uncertain processes, their $\alpha$-path are, respectively, given by formulas (36) and (37). According to Theorem 1, the uncertain variable

$$
\left(K-\left(X_{T}-Y_{T}\right)\right)^{+} \text {, }
$$

has the $\alpha$-path

$$
\left(K-\left(X_{T}^{-1}(1-\alpha)-Y_{T}^{-1}(\alpha)\right)\right)^{+} .
$$

So, the expectation of the uncertain variable $\left(K-\left(X_{T}-Y_{T}\right)\right)^{+}$is given by the following equation:

$$
E\left[\left(K-\left(X_{T}-Y_{T}\right)\right)^{+}\right]=\int_{0}^{1}\left(K-\left(X_{T}^{-1}(1-\alpha)-Y_{T}^{-1}(\alpha)\right)\right)^{+} \mathrm{d} \alpha .
$$

Thus, the price of the European spread put option is $f_{\text {Eput }}=E\left[\exp \left(-\int_{0}^{T} r_{t} \mathrm{~d} t\right)\right] \int_{0}^{1}\left(K-\left(X_{T}^{-1}(1-\alpha)-Y_{T}^{-1}(\alpha)\right)\right)^{+} \mathrm{d} \alpha$.

The pricing formula of the European spread put option is also derived.

From Theorem 9, the algorithm designed for calculating European spread put option price is the same as the procedure for calculating European spread call option price. Now, we will list the main procedures to calculate European spread put option price. First, we will calculate the expectation of $\exp \left(-\int_{0}^{T} r_{t} \mathrm{~d} t\right)$.

Step 0: set $t_{j}=j T / M, k=1,2, \ldots, L, j=1,2, \ldots, M$, where $L$ and $M$ are two large numbers.

Step 1: set $k=0$.

Step 2: set $k \longleftarrow k+1$.

Step 3: set $j=0$.

Step 4: set $j \longleftarrow j+1$.

Step 5: calculate the value of the stochastic interest rate at the time $t_{j}$ :

$$
r_{t_{j}}^{k}=r_{t_{j-1}}^{k}+a\left(b-r_{t_{j-1}}^{k}\right)\left(t_{j}-t_{j-1}\right)+\sigma_{0} \sqrt{r_{t_{j-1}}^{k}} \sqrt{t_{j}-t_{j-1}} \varepsilon_{j}^{k},
$$

where $\varepsilon_{j}^{k} \sim N(0,1)$ are generated by stochastic simulations.

Step 6: calculate the discount rate:

$$
\exp \left(-\int_{0}^{T} r_{t}^{k} \mathrm{~d} t\right) \longleftarrow \exp \left(-\frac{T}{M} \sum_{j=1}^{M} r_{t_{j}}^{k}\right) .
$$

Step 7: calculate the approximated value of $E\left[\exp \left(-\int_{0}^{T} r_{t} \mathrm{~d} t\right)\right]$ : 


$$
E\left[\exp \left(-\int_{0}^{T} r_{t} \mathrm{~d} t\right)\right] \longleftarrow \frac{1}{L} \sum_{k=1}^{L} \exp \left(-\frac{T}{M} \sum_{j=1}^{M} r_{t_{j}}^{k}\right)
$$

Second, we will calculate $E\left[\left(K-\left(X_{T}-Y_{T}\right)\right)^{+}\right]$.

Step 0: set $\alpha_{i}=i / N, i=1,2, \ldots, N-1$, where $N$ is a large number.

Step 1: set $i=0$.

Step 2: set $i \longleftarrow i+1$.

Step 3: calculate the inverse uncertainty distribution of the stock processes:

$$
\begin{aligned}
X_{T}^{-1}\left(1-\alpha_{i}\right)= & \exp \left(\exp \left(-\mu_{1} c T\right) \ln X_{0}+\frac{1}{c}\left(1-\exp \left(-\mu_{1} c T\right)\right)\right. \\
& \left.\left(1+\frac{\sqrt{3} \sigma_{1}}{\mu_{1} \pi} \ln \frac{1-\alpha_{i}}{\alpha_{i}}\right)\right) \\
Y_{T}^{-1}\left(\alpha_{i}\right)= & Y_{0} \exp \left(\mu_{2} T+\frac{\sqrt{3} \sigma_{2}}{\pi} T \ln \frac{\alpha_{i}}{1-\alpha_{i}}\right) .
\end{aligned}
$$

Step 4: set

$$
\gamma^{\alpha_{i}} \longleftarrow \max \left(K-\left(X_{T}^{-1}\left(1-\alpha_{i}\right)-Y_{T}^{-1}\left(\alpha_{i}\right)\right), 0\right) .
$$

If $i<N-1$, then return to Step 2 .

Step 5: calculate the expectation of $\left(K-\left(X_{T}-Y_{T}\right)\right)^{+}$:

$$
E\left[\left(K-\left(X_{T}-Y_{T}\right)\right)^{+}\right] \longleftarrow \frac{1}{N-1} \sum_{i=1}^{N-1} \gamma^{\alpha_{i}} .
$$

In the final procedure, the price of the European spread put option is derived by using Theorem 9 .

Example 2. Assume that the parameters of interest rate are $r_{0}=0.08, a=0.05, \sigma_{0}=0.04, b=2$, the parameters of stock price $X$ are $X_{0}=5, \mu_{1}=\sqrt{3}, \sigma_{1}=0.3, c=0.3$, and the parameters of stock price $Y$ are $Y_{0}=4, \mu_{2}=1, \sigma_{2}=0.2$. Then, the price of the European spread put option with maturity date $T=1$ and strike price $K=1$ is $f_{\text {Eput }}=1.9059$.

\section{Conclusions}

In this paper, we discuss the pricing of European spread options with the floating interest rate by chance theory. Some numerical algorithms are designed to calculate the price, and numerical simulations of spread options are given. Future research can think about some other multiasset option pricing problems.

\section{Data Availability}

All data used to support the findings of this study are included within this article.

\section{Conflicts of Interest}

The authors declare that they have no conflicts of interest.

\section{Acknowledgments}

This research was funded by the Youth Project of $\mathrm{Hu}-$ manities and Social Sciences of Ministry of Education (Grant nos. 19YJCZH251 and 20YJCZH245) and the Scientific Research Program of Tianjin Education Commission (Grant no. 2018KJ113).

\section{References}

[1] F. Black and M. Scholes, "The pricing of options and corporate liabilities," Journal of Political Economy, vol. 81, no. 3, pp. 637-654, 1973.

[2] R. C. Merton, "Theory of rational option pricing," The Bell Journal of Economics and Management Science, vol. 4, no. 1, pp. 141-183, 1973.

[3] J. Hull and A. White, "The pricing of options on assets with stochastic volatilities," The Journal of Finance, vol. 42, no. 2, pp. 281-300, 1987.

[4] S. L. Heston, "A closed-form solution for options with stochastic volatility with applications to bond and currency options," Review of Financial Studies, vol. 6, no. 2, pp. 327-343, 1993.

[5] B. Liu, Uncertainty Theory, Springer, Berlin, Germany, 2007.

[6] B. Liu, "Fuzzy process, hybrid process and uncertain process," Journal of Uncertain Systems, vol. 2, no. 1, pp. 3-16, 2008.

[7] B. Liu, "Some research problems in uncertainty theory," Journal of Uncertain Systems, vol. 3, no. 1, pp. 3-10, 2009.

[8] X. Chen, "American option pricing formula for uncertain financial market," International Journal of Operations Research, vol. 8, no. 2, pp. 32-37, 2011.

[9] Z. Zhang and W. Liu, "Geometric average Asian option pricing for uncertain financial market," Journal of Uncertain Systems, vol. 8, no. 4, pp. 317-320, 2014.

[10] X. Chen and J. Gao, "Uncertain term structure model of interest rate," Soft Computing, vol. 17, no. 4, pp. 597-604, 2013.

[11] K. Yao, "A no-arbitrage theorem for uncertain stock model," Fuzzy Optimization and Decision Making, vol. 14, no. 2, pp. 227-242, 2015.

[12] Z. Zhang, D. A. Ralescu, and W. Liu, "Valuation of interest rate ceiling and floor in uncertain financial market," Fuzzy Optimization and Decision Making, vol. 15, no. 2, pp. 139-154, 2016.

[13] Y. Sun and T. Su, "Mean-reverting stock model with floating interest rate in uncertain environment," Fuzzy Optimization and Decision Making, vol. 16, no. 2, pp. 235-255, 2017.

[14] Y. Gao, X. Yang, and Z. Fu, "Lookback option pricing problem of uncertain exponential Ornstein-Uhlenbeck model," Soft Computing, vol. 22, no. 17, pp. 5647-5654, 2018.

[15] R. Gao, "Stability in mean for uncertain differential equation with jumps," Applied Mathematics and Computation, vol. 346, pp. 15-22, 2019.

[16] Z. Lu and Y. Zhu, "Numerical approach for solution to an uncertain fractional differential equation," Applied Mathematics and Computation, vol. 343, pp. 137-148, 2019.

[17] Z. Li, Y.-J. Liu, and W.-G. Zhang, "Quasi-closed-form solution and numerical method for currency option with uncertain volatility model," Soft Computing, pp. 1-17, 2020. 
[18] W. Yu, P. Guo, Q. Wang et al., "On a periodic capital injection and barrier dividend strategy in the compound Poisson risk model," Mathematics, vol. 8, no. 4, p. 511, 2020.

[19] W. Yu, F. Wang, Y. Huang, and H. Liu, "Social optimal mean field control problem for population growth model," Asian Journal of Control, vol. 21, no. 4, pp. 1-8, 2019.

[20] L. Zhang and Y. Sun, "Power options pricing in uncertain environment," Acta Scientiarum Naturalium Universitatis Nankaiensis, vol. 53, no. 2, pp. 1-6, 2020.

[21] B. Liu, Uncertainty Theory, Springer, Berlin, Germany, 2015.

[22] B. Liu, Uncertainty Theory: A Branch of Mathematics for Modeling Human Uncertainty, Springer, Berlin, Germany, 2010.

[23] K. Yao and X. Chen, "A numerical method for solving uncertain differential equations," Journal of Intelligent \& Fuzzy Systems, vol. 25, no. 3, pp. 825-832, 2013.

[24] Y. Liu, "Uncertain random variables: a mixture of uncertainty and randomness," Soft Computing, vol. 17, no. 4, pp. 625-634, 2013.

[25] Y. Liu, "Uncertain random programming with applications," Fuzzy Optimization and Decision Making, vol. 12, no. 2, pp. 153-169, 2013.

[26] L. Dai, Z. Fu, and Z. Huang, "Option pricing formulas for uncertain financial market based on the exponential Ornstein-Uhlenbeck model," Journal of Intelligent Manufacturing, vol. 28, no. 3, pp. 597-604, 2017. 